\title{
Coming to order: Adsorption and structure of nonionic polymer at the oil/water interface as influenced by cationic and anionic surfactants Supporting Information
}

\author{
Rebecca M. Altman, Geraldine L. Richmond* \\ Department of Chemistry and Biochemistry, University of Oregon, Eugene, Oregon, 97403 \\ United States
}

Table of Contents:

Figure 3 spectral fit parameters: pgs. 2-3

Figure 5 spectral fit parameters: pg. 4

Figure 6 spectral fit parameters: pg. 4

Figure 7 spectral inset fit parameters: pg. 5

Figure 8 spectral fit parameters: pgs. 6-8

Supplementary VSF spectra: pg. 9

*Corresponding author, email: richmond@uoregon.edu, phone: (541) 346-4635 


\section{SUPPLEMENTAL INFORMATION:}

Variables in the following tables correspond to the VSF fit equation given in Equation 3.

\section{FIGURE 3 Spectral fits:}

\begin{tabular}{|c|c|c|c|c|}
\hline \multicolumn{2}{|c|}{$\begin{array}{c}\text { PAM + } 0.015 \text { mM d-DTAB } \\
(S S P) \\
\end{array}$} & \multirow{2}{*}{$\begin{array}{r}0.015 \mathrm{mM} \text { PAM } \\
0.06 \pm 0.01\end{array}$} & \multirow{2}{*}{$\begin{array}{c}4.2 \mathrm{mM} \text { PAM } \\
0.096 \pm 0.009\end{array}$} & \multirow{2}{*}{ 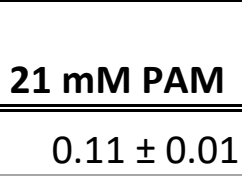 } \\
\hline \multirow{5}{*}{$\begin{array}{l}\text { Peak } 1 \\
\text { (C-H stretch) }\end{array}$} & Amplitude & & & \\
\hline & Phase & 0 & 0 & 0 \\
\hline & Lorentzian & 2 & 2 & 2 \\
\hline & Peak Position & $2875 \pm 3$ & $2872 \pm 2$ & $2871 \pm 2$ \\
\hline & Gaussian & $15 \pm 4$ & $15 \pm 2$ & $15 \pm 3$ \\
\hline \multirow{5}{*}{$\begin{array}{c}\text { Peak } 2 \\
\left(\mathrm{CH}_{2} \text { symmetric }\right. \\
\text { stretch })\end{array}$} & Amplitude & $0.319 \pm 0.003$ & $0.450 \pm 0.003$ & $0.458 \pm 0.004$ \\
\hline & Phase & 0 & 0 & 0 \\
\hline & Lorentzian & 2 & 2 & 2 \\
\hline & Peak Position & $2935.5 \pm 0.3$ & $2935.7 \pm 0.2$ & $2935.2 \pm 0.2$ \\
\hline & Gaussian & $14.4 \pm 0.3$ & $14.1 \pm 0.2$ & $14.0 \pm 0.3$ \\
\hline
\end{tabular}

Table S1: Fit parameters for mixed system traces in Figure 3a. All solutions consisted of PAM $(0.015,4.2$, and $21 \mathrm{mM})$ with $0.015 \mathrm{mM}$ d-DTAB in $\mathrm{D}_{2} \mathrm{O}$. Peaks 1 and 2 correspond to the alpha carbon $\mathrm{CH}$ stretch and the $\mathrm{CH}_{2}$ symmetric stretch $(\mathrm{d}+)$ from the PAM backbone, respectively. 


\begin{tabular}{|c|c|c|}
\hline \multicolumn{3}{|c|}{$0.015 \mathrm{mM}$ d-DTAB (SSP) } \\
\hline \multirow{5}{*}{$\begin{array}{c}\text { Peak } 1 \\
\text { (Coordinated } \\
\mathrm{D}_{2} \mathrm{O} \text { peak } 1 \text { ) }\end{array}$} & Amplitude & $0.92 \pm 0.02$ \\
\hline & Phase & 3.14 \\
\hline & Lorentzian & 2 \\
\hline & Peak Position & $2576 \pm 4$ \\
\hline & Gaussian & $47 \pm 3$ \\
\hline \multirow{5}{*}{$\begin{array}{c}\text { Peak } 2 \\
\text { (Coordinated } \\
\mathrm{D}_{2} \mathrm{O} \text { peak } 2 \text { ) }\end{array}$} & Amplitude & $0.42 \pm 0.02$ \\
\hline & Phase & 0 \\
\hline & Lorentzian & 2 \\
\hline & Peak Position & $2719 \pm 1$ \\
\hline & Gaussian & $18 \pm 1$ \\
\hline \multirow{5}{*}{$\begin{array}{c}\text { Peak } 3 \\
\text { (Free OD) }\end{array}$} & Amplitude & $0.11 \pm 0.02$ \\
\hline & Phase & 0 \\
\hline & Lorentzian & 2 \\
\hline & Peak Position & $2682 \pm 1$ \\
\hline & Gaussian & $15 \pm 3$ \\
\hline
\end{tabular}

Table S2: Fit parameters for the d-DTAB in $\mathrm{D}_{2} \mathrm{O}$ (grey) trace in Figure 3a. Peaks 1 and 2 correspond to coordinated water modes and Peak 3 corresponds to the free OD mode.

\begin{tabular}{|c|c|c|c|c|}
\hline \multicolumn{2}{|c|}{$\begin{array}{c}\text { PAM + } 0.015 \mathrm{mM} d-\mathrm{DTAB} \\
\text { (SPS) }\end{array}$} & \multirow{2}{*}{$\begin{array}{r}0.015 \mathrm{mM} \text { PAM } \\
1.71 \pm 0.09\end{array}$} & \multirow{2}{*}{$\begin{array}{r}4.2 \mathrm{mM} \text { PAM } \\
1.60 \pm 0.09\end{array}$} & \multirow{2}{*}{$21 \mathrm{mM}$ PAM } \\
\hline \multirow{5}{*}{$\begin{array}{c}\text { Peak } 1 \\
\text { (C-H stretch) }\end{array}$} & Amplitude & & & \\
\hline & Phase & 0 & 0 & 0 \\
\hline & Lorentzian & 2 & 2 & 2 \\
\hline & Peak Position & $2875 \pm 3$ & $2873 \pm 3$ & $2870 \pm 3$ \\
\hline & Gaussian & $63 \pm 14$ & $63 \pm 13$ & $52 \pm 7$ \\
\hline \multirow{5}{*}{$\begin{array}{c}\text { Peak } 2 \\
\left(\mathrm{CH}_{2} \text { symmetric }\right. \\
\text { stretch })\end{array}$} & Amplitude & $0.12 \pm 0.2$ & $1.2 \pm 0.2$ & $1.07 \pm 0.07$ \\
\hline & Phase & 0 & 0 & 0 \\
\hline & Lorentzian & 2 & 2 & 2 \\
\hline & Peak Position & $2959 \pm 2$ & $2957 \pm 2$ & $2957 \pm 1$ \\
\hline & Gaussian & $32 \pm 3$ & $30 \pm 3$ & $28 \pm 2$ \\
\hline \multirow{2}{*}{ Nonresonant } & Amplitude & $1.09 \pm 0.05$ & $1.07 \pm 0.05$ & $1.08 \pm 0.04$ \\
\hline & Phase & 3.14 & 3.14 & 3.14 \\
\hline
\end{tabular}

Table S3: Fit parameters for mixed system traces in Figure 3b. All solutions consisted of PAM $(0.015,4.2$, and $21 \mathrm{mM})$ with $0.015 \mathrm{mM}$ d-DTAB in $\mathrm{D}_{2} \mathrm{O}$. Peaks 1 and 2 correspond to the alpha carbon $\mathrm{CH}$ stretch and the $\mathrm{CH}_{2}$ asymmetric stretch (d-) from the PAM backbone, respectively. The elevated background in the spectra is fit to a nonresonant signal. 
FIGURE 5 Spectral fits:

\begin{tabular}{|c|l||r|r|r|}
\hline $\begin{array}{c}\text { PAM + 0.015 mM d-DTAB } \\
(\text { SSP) }\end{array}$ & & & \\
\hline \hline \multirow{5}{*}{ C=0 peak } & Amplitude & $1.51 \pm 0.09$ & $2.2 \pm 0.1$ & $1.85 \pm 0.08$ \\
\cline { 2 - 5 } & Phase & 0 & 0 & 0 \\
\cline { 2 - 5 } & Lorentzian & 5 & 5 & 41 mM PAM \\
\cline { 2 - 5 } & Peak Position & $1650.4 \pm 0.8$ & $1651.1 \pm 0.6$ & $1649.6 \pm 0.5$ \\
\cline { 2 - 5 } & Gaussian & $12 \pm 1$ & $10.3 \pm 0.8$ & $10.0 \pm 0.7$ \\
\hline
\end{tabular}

Table S4: Fit parameters for mixed system traces of the carbonyl region in Figure 5. All solutions consisted of PAM $(0.015 \mathrm{mM}, 4.2$, and $21 \mathrm{mM})$ with $0.015 \mathrm{mM}$ d-DTAB in $\mathrm{D}_{2} \mathrm{O}$.

FIGURE 6 Spectral fits:

\begin{tabular}{|c|c|c|c|c|}
\hline \multicolumn{2}{|c|}{$\begin{array}{c}\text { PAM + } 0.001 \mathrm{mM} \text { d-CTAB } \\
(\mathrm{SSP}) \\
\end{array}$} & \multirow{2}{*}{$\frac{0.015 \text { mM PAM }}{0.051 \pm 0.007}$} & \multirow{2}{*}{$\frac{4.2 \mathrm{mM} \text { PAM }}{0.07 \pm 0.008}$} & \multirow{2}{*}{ 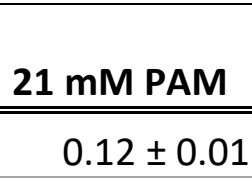 } \\
\hline \multirow{5}{*}{$\begin{array}{c}\text { Peak } 1 \\
\text { (C-H stretch) }\end{array}$} & Amplitude & & & \\
\hline & Phase & 0 & 0 & 0 \\
\hline & Lorentzian & 2 & 2 & 2 \\
\hline & Peak Position & $2879 \pm 3$ & $2875 \pm 2$ & $2873 \pm 2$ \\
\hline & Gaussian & $20 \pm 4$ & $15 \pm 2$ & $12 \pm 2$ \\
\hline \multirow{5}{*}{$\begin{array}{c}\text { Peak } 2 \\
\left(\mathrm{CH}_{2} \text { symmetric }\right. \\
\text { stretch })\end{array}$} & Amplitude & $0.266 \pm 0.002$ & $0.413 \pm 0.002$ & $0.396 \pm 0.004$ \\
\hline & Phase & 0 & 0 & 0 \\
\hline & Lorentzian & 2 & 2 & 2 \\
\hline & Peak Position & $2937.2 \pm 0.3$ & $2937.1 \pm 0.2$ & $2934.0 \pm 0.3$ \\
\hline & Gaussian & $15.7 \pm 0.3$ & $15.4 \pm 0.2$ & $15.4 \pm 0.3$ \\
\hline
\end{tabular}

Table S5: Fit parameters for mixed system traces in Figure 6. All solutions consisted of PAM $(0.015,4.2$, and $21 \mathrm{mM})$ with $0.001 \mathrm{mM}$ d-CTAB in $\mathrm{D}_{2} \mathrm{O}$. Peaks 1 and 2 correspond to the alpha carbon $\mathrm{CH}$ stretch and the $\mathrm{CH}_{2}$ symmetric stretch $(\mathrm{d}+)$ from the PAM backbone, respectively. 
FIGURE 7 Spectral fits:

\begin{tabular}{|c|c|c|c|}
\hline \multicolumn{2}{|c|}{$\begin{array}{c}\text { 4.2mM PAM + } 0.015 \mathrm{mM} \\
\mathrm{d}-\mathrm{DTAB}(\mathrm{SSP}) \\
\end{array}$} & \multirow{2}{*}{$\frac{@ \mathbf{t}=\mathbf{t}_{\mathbf{o}}}{0.07 \pm 0.008}$} & \multirow{2}{*}{$\frac{@ \mathrm{t}>\mathrm{t}_{\mathrm{eq}}}{0.07 \pm 0.008}$} \\
\hline \multirow{5}{*}{$\begin{array}{c}\text { Peak } 1 \\
\text { (C-H stretch) }\end{array}$} & Amplitude & & \\
\hline & Phase & 0 & 0 \\
\hline & Lorentzian & 2 & 2 \\
\hline & Peak Position & $2875 \pm 2$ & $2874 \pm 2$ \\
\hline & Gaussian & $17 \pm 3$ & $16 \pm 2$ \\
\hline \multirow{5}{*}{$\begin{array}{c}\text { Peak } 2 \\
\left(\mathrm{CH}_{2} \text { symmetric }\right. \\
\text { stretch })\end{array}$} & Amplitude & $0.357 \pm 0.002$ & $0.363 \pm 0.002$ \\
\hline & Phase & 0 & 0 \\
\hline & Lorentzian & 2 & 2 \\
\hline & Peak Position & $2936.4 \pm 0.2$ & $2936.0 \pm 0.2$ \\
\hline & Gaussian & $14.6 \pm 0.2$ & $14.1 \pm 0.2$ \\
\hline
\end{tabular}

Table S6: Fit parameters for the mixed system spectral insets in Figure 7. All solutions consisted of $4.2 \mathrm{mM}$ PAM with $0.015 \mathrm{mM}$ d-DTAB in $\mathrm{D}_{2} \mathrm{O}$. Peaks 1 and 2 correspond to the alpha carbon $\mathrm{CH}$ stretch and the $\mathrm{CH}_{2}$ symmetric stretch $(\mathrm{d}+)$ from the PAM backbone, respectively. Comparison of the VSF peak fit intensities from $t_{0}$ to $t_{\text {eq }}$ show the spectra of polyacrylamide is unchanging over time. 
FIGURE 8 Spectral Fits:

\begin{tabular}{|c|c|c|c|c|}
\hline \multicolumn{2}{|c|}{$\begin{array}{c}\text { PAM + } 0.015 \mathrm{mM} \text { d-SDS } \\
\text { (SSP) }\end{array}$} & \multirow{2}{*}{$\begin{array}{r}0.015 \mathrm{mM} \text { PAM } \\
0.9 \pm 0.1\end{array}$} & \multirow{2}{*}{$\begin{array}{r}4.2 \mathrm{mM} \text { PAM } \\
0.7 \pm 0.1\end{array}$} & \multirow{2}{*}{$\frac{21 \mathrm{mM} \text { PAM }}{0.7 \pm 0.2}$} \\
\hline \multirow{5}{*}{$\begin{array}{c}\text { Peak } 1 \\
\text { (Coordinated } \\
\left.\quad \mathrm{D}_{2} \mathrm{O}\right)\end{array}$} & Amplitude & & & \\
\hline & Phase & 0 & 0 & 0 \\
\hline & Lorentzian & 5 & 5 & 5 \\
\hline & Peak Position & $2485 \pm 6$ & $2484 \pm 25$ & $2486 \pm 48$ \\
\hline & Gaussian & $80 \pm 6$ & $96 \pm 4$ & $110 \pm 7$ \\
\hline \multirow{5}{*}{$\begin{array}{l}\text { Peak } 2 \\
\text { (Free OD) }\end{array}$} & Amplitude & $0.5 \pm 0.1$ & $0.42 \pm 0.08$ & $0.5 \pm 0.2$ \\
\hline & Phase & 0 & 0 & 0 \\
\hline & Lorentzian & 12 & 12 & 12 \\
\hline & Peak Position & $2716 \pm 1$ & $2717 \pm 2$ & $2719 \pm 2$ \\
\hline & Gaussian & $8 \pm 2$ & $10 \pm 2$ & $8 \pm 3$ \\
\hline \multirow{5}{*}{$\begin{array}{l}\text { Peak } 3 \\
\text { (Background } \\
\text { peak 1) }\end{array}$} & Amplitude & $0.09 \pm 0.03$ & $0.1 \pm 0.04$ & $0.10 \pm 0.04$ \\
\hline & Phase & 0 & 0 & 0 \\
\hline & Lorentzian & 5 & 5 & 5 \\
\hline & Peak Position & $2789 \pm 2$ & $2790 \pm 3$ & $2791 \pm 3$ \\
\hline & Gaussian & $40 \pm 8$ & $43 \pm 10$ & $44 \pm 10$ \\
\hline \multirow{5}{*}{$\begin{array}{l}\text { Peak } 4 \\
\text { (Background } \\
\text { peak 2) }\end{array}$} & Amplitude & $0.067 \pm 0.009$ & $0.07 \pm 0.02$ & $0.08 \pm 0.03$ \\
\hline & Phase & 0 & 0 & 0 \\
\hline & Lorentzian & 5 & 5 & 5 \\
\hline & Peak Position & $2871 \pm 31$ & $2872 \pm 47$ & $2878 \pm 43$ \\
\hline & Gaussian & $111 \pm 31$ & $112 \pm 46$ & $115 \pm 46$ \\
\hline \multirow{5}{*}{$\begin{array}{c}\text { Peak } 5 \\
\left(\mathrm{CH}_{2} \text { symmetric }\right. \\
\text { stretch })\end{array}$} & Amplitude & $0.08 \pm 0.01$ & $0.09 \pm 0.01$ & $0.07 \pm 0.01$ \\
\hline & Phase & 3.14 & 3.14 & 3.14 \\
\hline & Lorentzian & 2 & 2 & 2 \\
\hline & Peak Position & $2932 \pm 2$ & $2931 \pm 2$ & $2928 \pm 3$ \\
\hline & Gaussian & $13 \pm 3$ & $16 \pm 3$ & $20 \pm 5$ \\
\hline
\end{tabular}

Table S7: Fit parameters for mixed system traces in Figure 8a. All solutions consisted of PAM $(0.015,4.2$, and $21 \mathrm{mM})$ with $0.015 \mathrm{mM}$ d-SDS in $\mathrm{D}_{2} \mathrm{O}$. Peaks 1 and 2 correspond to the coordinated water and free OD modes, respectively. Peaks 3 and 4 fit the broad signal from $\sim 2750-3050 \mathrm{~cm}^{-1}$, characterizing the background signal from the sulfate headgroup of d-SDS. Peak 5 is assigned to the $d+$ stretch from the PAM backbone. 


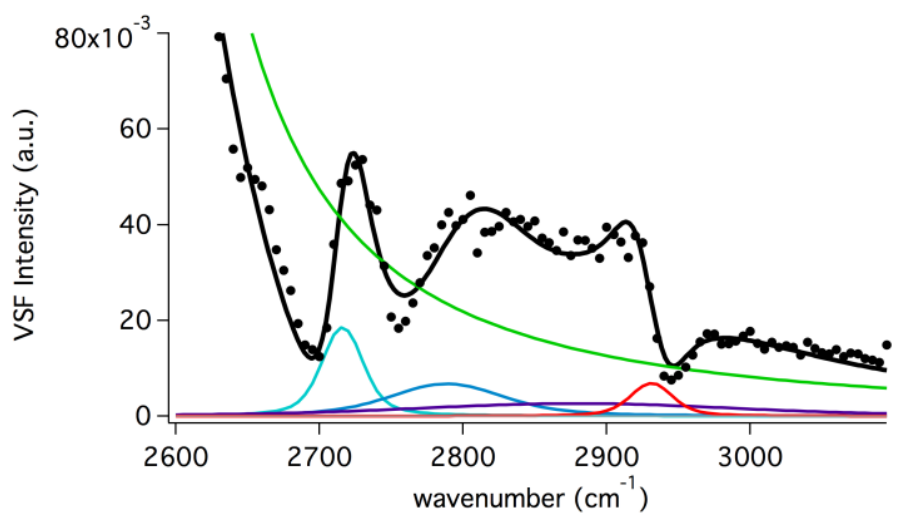

Figure S1: The underlying five peaks that contribute the full fit for $4.2 \mathrm{mM}$ PAM with $0.015 \mathrm{mM}$ $\mathrm{d}$-SDS in $\mathrm{D}_{2} \mathrm{O}$ in Figure $8 \mathrm{a}$ (black trace shown here). The coordinated $\mathrm{D}_{2} \mathrm{O}$, Free OD, and two background peaks are displayed as the green, light blue, and dark blue and purple traces, respectively. The red peak is the PAM asymmetric $\mathrm{CH}_{2}$ stretch (also shown in Figure 8a), which is responsible for the interference feature observed in all the mixed system spectra in Figure 8 a.

\begin{tabular}{|c|c|c|}
\hline \multicolumn{3}{|c|}{$0.015 \mathrm{mM}$ d-SDS (SSP) } \\
\hline \multirow{5}{*}{$\begin{array}{c}\text { Peak } 1 \\
\text { (Coordinated } \\
\left.\mathrm{D}_{2} \mathrm{O}\right)\end{array}$} & Amplitude & $0.9 \pm 0.2$ \\
\hline & Phase & 0 \\
\hline & Lorentzian & 5 \\
\hline & Peak Position & $2480 \pm 30$ \\
\hline & Gaussian & $100 \pm 4$ \\
\hline \multirow{5}{*}{$\begin{array}{c}\text { Peak } 2 \\
\text { (Free OD) }\end{array}$} & Amplitude & $0.62 \pm 0.06$ \\
\hline & Phase & 0 \\
\hline & Lorentzian & 12 \\
\hline & Peak Position & $2719 \pm 2$ \\
\hline & Gaussian & $11 \pm 1$ \\
\hline \multirow{5}{*}{$\begin{array}{c}\text { Peak } 3 \\
\text { (Background } \\
\text { peak 1) }\end{array}$} & Amplitude & $0.10 \pm 0.01$ \\
\hline & Phase & 0 \\
\hline & Lorentzian & 5 \\
\hline & Peak Position & $2781 \pm 3$ \\
\hline & Gaussian & $40 \pm 8$ \\
\hline \multirow{5}{*}{$\begin{array}{c}\text { Peak } 4 \\
\text { (Background } \\
\text { peak 2) }\end{array}$} & Amplitude & $0.14 \pm 0.02$ \\
\hline & Phase & 0 \\
\hline & Lorentzian & 5 \\
\hline & Peak Position & $2880 \pm 9$ \\
\hline & Gaussian & $112 \pm 14$ \\
\hline
\end{tabular}

Table S8: Fit parameters for the d-SDS in $\mathrm{D}_{2} \mathrm{O}$ (grey) trace in Figure 8a. Peaks 1 and 2 correspond to the coordinated water and free OD modes, respectively. Peaks 3 and 4 fit the broad signal from $\sim 2750-3050 \mathrm{~cm}^{-1}$, characterizing the background signal from the sulfate headgroup of SDS. 


\begin{tabular}{|c|c|c|c|}
\hline \multicolumn{2}{|c|}{$\begin{array}{c}0.015 \mathrm{mM} \mathrm{d}-\mathrm{SDS}+1 \mathrm{M} \mathrm{NaCl} \\
(\mathrm{SSP})\end{array}$} & \multirow{2}{*}{$\frac{0 \mathrm{mM} \text { PAM }}{0.07 \pm 0.01}$} & \multirow{2}{*}{$\begin{array}{r}4.2 \mathrm{mM} \text { PAM } \\
0.080 \pm 0.007\end{array}$} \\
\hline \multirow{5}{*}{$\begin{array}{c}\text { Peak } 1 \\
\text { (Coordinated } \\
\mathrm{D}_{2} \mathrm{O} \text { peak } 1 \text { ) }\end{array}$} & Amplitude & & \\
\hline & Phase & 0 & 0 \\
\hline & Lorentzian & 5 & 5 \\
\hline & Peak Position & $2646 \pm 4$ & $2640 \pm 8$ \\
\hline & Gaussian & $60 \pm 10$ & $75 \pm 22$ \\
\hline \multirow{5}{*}{$\begin{array}{c}\text { Peak } 2 \\
\text { (Coordinated } \\
\mathrm{D}_{2} \mathrm{O} \text { peal } 2 \text { ) }\end{array}$} & Amplitude & $0.27 \pm 0.01$ & $0.38 \pm 0.01$ \\
\hline & Phase & 0 & 0 \\
\hline & Lorentzian & 5 & 5 \\
\hline & Peak Position & $2488 \pm 12$ & $2490 \pm 8$ \\
\hline & Gaussian & $88 \pm 11$ & $66 \pm 8$ \\
\hline \multirow{5}{*}{$\begin{array}{l}\text { Peak } 3 \\
\text { (Free OD) }\end{array}$} & Amplitude & $0.11 \pm 0.03$ & $0.1 \pm 0.1$ \\
\hline & Phase & 0 & 0 \\
\hline & Lorentzian & 12 & 12 \\
\hline & Peak Position & $2720 \pm 2$ & $2719 \pm 4$ \\
\hline & Gaussian & $11 \pm 5$ & $8 \pm 11$ \\
\hline \multirow{5}{*}{$\begin{array}{l}\text { Peak } 4 \\
\text { (Background } \\
\text { peak 1) }\end{array}$} & Amplitude & $0.03 \pm 0.01$ & $0.04 \pm 0.02$ \\
\hline & Phase & 0 & 0 \\
\hline & Lorentzian & 5 & 5 \\
\hline & Peak Position & $2785 \pm 8$ & $2790 \pm 9$ \\
\hline & Gaussian & $53 \pm 18$ & $43 \pm 19$ \\
\hline \multirow{5}{*}{$\begin{array}{l}\text { Peak } 5 \\
\text { (Background } \\
\text { peak 2) }\end{array}$} & Amplitude & $0.067 \pm 0.004$ & $0.056 \pm 0.004$ \\
\hline & Phase & 0 & 0 \\
\hline & Lorentzian & 5 & 5 \\
\hline & Peak Position & $2906 \pm 18$ & $2909 \pm 21$ \\
\hline & Gaussian & $174 \pm 35$ & $123 \pm 37$ \\
\hline
\end{tabular}

Table S9: Fit parameters for mixed system traces in Figure 8b. All solutions contain $0.015 \mathrm{mM}$ dSDS and $1 \mathrm{M} \mathrm{NaCl}$ in $\mathrm{D}_{2} \mathrm{O}$, with either $0 \mathrm{mM}$ or $4.2 \mathrm{mM}$ PAM. Peaks 1 and 2 correspond to the two coordinated water modes, and Peak 3 corresponds to the Free OD mode. Peaks 4 and 5 are two background peaks, fitting the broad signal from $\sim 2750-3050 \mathrm{~cm}^{-1}$ to characterize the background signal from the sulfate headgroup of $d-S D S$. 
SUPPLEMENTARY VSF SPECTRA:

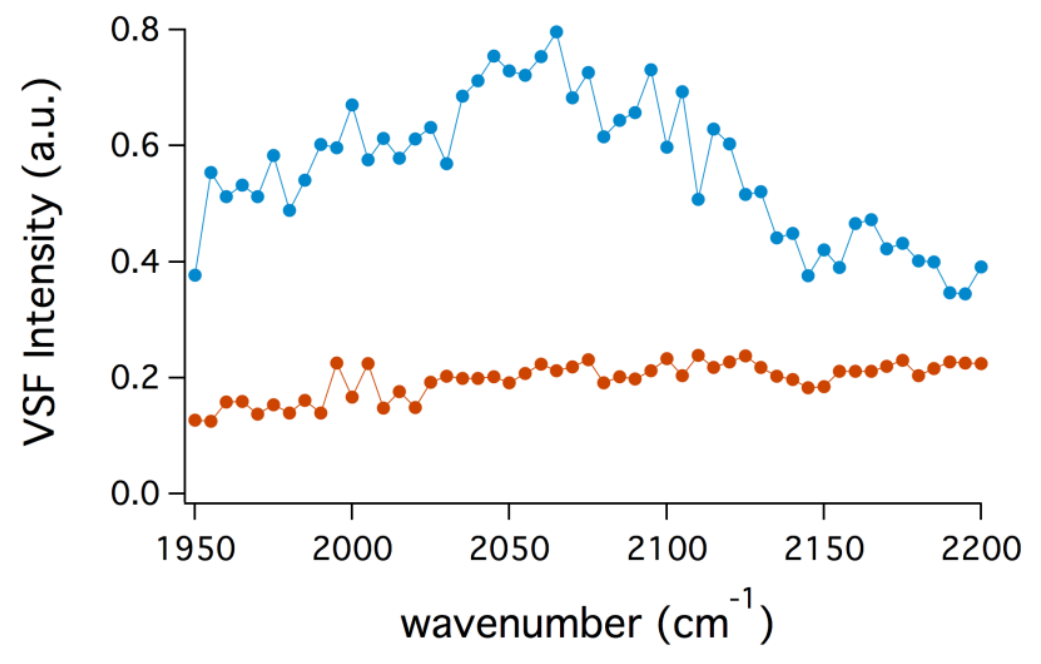

Figure S2: Spectra in SSP polarization combination of $0.015 \mathrm{mM}$ d-DTAB in solvents $\mathrm{H}_{2} \mathrm{O}$ (orange trace) and $\mathrm{D}_{2} \mathrm{O}$ (blue trace). Lines are guides to the eye. No C-D peaks from d-DTAB are observed around $2100 \mathrm{~cm}^{-1}$ in either spectra. At this concentration of surfactant, the C-D modes of the d-DTAB are hidden under the VSF signal from coordinated water molecules, which is induced by the surfactant's interfacial charge. Because $\mathrm{D}_{2} \mathrm{O}$ modes are redshifted to lower wavenumbers from the $\mathrm{H}_{2} \mathrm{O}$ modes, the surfactant solution containing $\mathrm{D}_{2} \mathrm{O}$ as the solvent (blue trace) has a higher VSF intensity. In either solvent, C-D modes of surfactant are not observed. 